\title{
Generation of an infectious clone of HuN4-F112, an attenuated live vaccine strain of porcine reproductive and respiratory syndrome virus
}

\author{
Shanrui Zhang, Yanjun Zhou, Yifeng Jiang, Guoxin Li, Liping Yan, Hai Yu and Guangzhi Tong*
}

\begin{abstract}
Background: Nowadays, PRRS has become one of the most economically important infectious diseases of pig worldwide. To better characterize and understand the molecular basis of PRRSV virulence determinants, it would be important to develop the infectious cDNA clones. In this regard, HuN4-F112, a live-attenuated North-Americantype PRRSV vaccine strain, could serve as an excellent model.

Results: In the study, genomic sequence of HuN4-F112, an attenuated vaccine virus derived from the highly pathogenic porcine reproductive and respiratory syndrome virus (PRRSV) HuN4 strain, was determined and its fulllength cDNA was cloned. Capped RNA was transcribed in vitro from the cDNA clone and transfected into BHK-21 cells. The supernatant from transfected monolayers were serially passaged in Marc- 145 cells. The rescued virus exhibited a similar growth pattern to its parental virus in Marc-145 cells with peak titers at $48 \mathrm{~h}$ post-infection.

Conclusion: In conclusion, we rescued virus from an infectious cDNA clone of attenuated vaccine. It is possible in the future that a new attenuated PRRSV vaccine with broader specificity and good immunogenicity can be designed in vitro via an infectious CDNA clone platform coupled with validated information on virulence determinants.
\end{abstract}

Keywords: Infectious clone, PRRSV, HuN4-F112

\section{Background}

Porcine reproductive and respiratory syndrome (PRRS) was first reported in late 1980s in North America and shortly thereafter in Europe. The disease is characterized by reproductive failure in late gestation in sows and respiratory symptoms in pigs of all ages [1-3]. Nowadays, PRRS has become one of the most economically important infectious diseases of pig worldwide [4,5]. The PRRS virus (PRRSV) is a small enveloped positive-strand RNA virus belonging to the family Arteriviridae in the order Nidovirales together with equine arteritis virus (EAV), simian hemorrhagic fever virus, and lactate dehydrogenase-elevating virus (LDV) of mice[6,7]. PRRSV is further classified into two distinct genotypes, the North American type and the European type $[8,9]$.

\footnotetext{
* Correspondence: gztong@shvri.ac.cn

Division of Swine Infectious Diseases, Shanghai Veterinary Research Institute, Chinese Academy of Agricultural Sciences, Shanghai 200241, China
}

Since May 2006, an atypical PRRS (also known as porcine high fever syndrome) has been pandemic in China. Several studies have confirmed that the causative agent of the outbreaks was highly pathogenic PRRSV (HP-PRRSV) and several HP-PRRSV strains were isolated [10-12]. The genome sequences of several strains representing PRRSV have been determined and infectious cDNA clones were developed [13,14]. However, few of these came from a strain with attenuated virulence. To better characterize and understand the molecular basis of PRRSV virulence determinants, it would be important to develop infectious clone of a virulence-attenuated strain. In this regard, HuN4-F112, a live-attenuated North-American-type PRRSV vaccine strain, could serve as an excellent model.

The present article describes the genomic sequence of a virulence-attenuated PRRSV strain HuN4-F112 and the successful rescuing of the virus from its infectious full-length cDNA clone. 


\section{Materials and methods}

Virus strain and cell line

HuN4-F112 is a virulence-attenuated North-Americantype PRRSV strain derived via consecutive passage in Marc-145 cells. This virus strain was maintained in our laboratory as a live-attenuated vaccine [15]. BHK-21 cells were used to rescue virus by transfection with in vitrotranscribed RNA. Marc-145 cells were used for virus rescue and subsequent experiments. Cells were maintained as previously described [10].

\section{RNA extraction and molecular cloning of viral genomic cDNA fragments}

Total RNAs were isolated from the supernatants of MARC-145 cells culture at $48 \mathrm{~h}$ post infection (PI) by using a QIAamp viral RNA kit (QIAGEN). The cDNA synthesis was performed with SupersciptIII reverse transcriptase (Invitrogen) and reverse primers of each fragment (Table 1). The PCR primers in Table 1 were designed mainly according to the sequence of PRRSV HuN4 strain with the GeneBank accession number of EF635006[10]. Six fragments (designated as A, B, C, D, E and F. Figure 1). spanning the full-length genome, were subsequently generated by PCR amplification with Platinum ${ }^{\circledR}$ Taq DNA polymerase. Each PCR product was cloned into pCR-Blunt II-TOPO vector (Invitrogen). Nucleotide sequencing of the clones was performed by Shanghai Auke Inc. (Shanhai, China) using an ABI 377 automatic sequencer. The sequence data were analyzed using the Lasergene Software Package (DNAstar Inc., USA).
Construction of full-length cDNA clone of HuN4-F112

The strategy for construction of full-length cDNA clone of HuN4-F112 is illustrated in Figure 1. A total of six fragments, covering the complete HuN4-F112 genome, were subsequently PCR amplified with Platinum ${ }^{\circledR} p f x$ DNA polymerase according to the manufacturer's protocol. The A at position 14680 was mutated to G by PCR to create a restriction enzyme site $M l u \mathrm{I}$, a translationally silent substitution, both for fragment $\mathrm{E}$ and $\mathrm{F}$ ligation and as a genetic marker to differentiate the cloned virus and the parental virus. The bacteriophage SP6 RNA polymerase promoter (underlined sequence) and two non-viral guanosine residues were engineered into primer F16 (Table 1.).

The vector, pBluescript II SK(+) (Stratagene) was modified, the T7 RNA promoter in the vector was changed into the SP6 RNA promoter by PCR mutagenesis. The fragment between the XhoI and NotI sites were replaced by a stuffer fragment, which was prepared as a synthetic gene containing the restriction enzyme sites as shown in Figure 1. The PCR-amplified fragments were gel purified, digested with enzymes as indicated in Figure 1, and cloned into the modified pBluescript II SK(+) vector. After each ligation step, the constructed plasmid was transformed into Escherichia coli DH5 $\alpha$ cells and grown overnight at $37^{\circ} \mathrm{C}$ in the presence of ampicillin. Over-lapped cDNA fragments were joined by using shared restriction sites (Figure 1). Finally, a full-length HuN4-F112 cDNA clone, pHuN4-F112, was obtained (Figure 1). The completely assembled full-length cDNA clone was sequenced.

Table 1 Primers used for amplification of the genome of PRRSV HuN4-F112

\begin{tabular}{|c|c|c|}
\hline Primer $^{\mathrm{a}}$ & Sequences $^{\mathrm{b}}\left(5^{\prime}-3^{\prime}\right)$ & Position in HuN4 \\
\hline \multicolumn{3}{|c|}{ For fragment $A$} \\
\hline F16(sp6) & CCGCTCGAGTTAATTAAATITAGGTGACACTATAGGATGACGTATAGGTGTT & $1-16$ \\
\hline R2355 & GTGATGAACCTCGTCACCTTGTGCAGGG & 2355-2382 \\
\hline \multicolumn{3}{|c|}{ For fragment B } \\
\hline F2300 & CTTTGGGCAAGGACTCGGT & $2300-2319$ \\
\hline R5914 & GATCCTGTGTGAACGCCGAC & $5914-5933$ \\
\hline \multicolumn{3}{|c|}{ For fragment $\mathrm{C}$} \\
\hline F5853 & CTTCTGCTTCACCGCGTGT & 5853-5871 \\
\hline R8825 & AAGAAGATTGGCGGCAAAC & $8825-8843$ \\
\hline \multicolumn{3}{|c|}{ For fragment $D$} \\
\hline F8764 & GCAGGTGCCTTGAAGCTGAT & $8764-8783$ \\
\hline R11910 & CTCATGCTGATGGCATTAGC & $11910-11929$ \\
\hline \multicolumn{3}{|c|}{ For fragment $\mathrm{E}$} \\
\hline F11851 & AGGACTGGGAGGATTACAAT & $11851-11870$ \\
\hline R14670 & CGGACGACAAACGCGTGGTTAT & $14670-14691$ \\
\hline \multicolumn{3}{|c|}{ For fragment $\mathrm{F}$} \\
\hline F14668 & TGATAACCACGCGTTTGTCGTC & $14668-14689$ \\
\hline R15313 & TATAGCGGCCGCATTTAAAT(T) ${ }_{32}$ AATTACGG & $15313-15320$ \\
\hline
\end{tabular}

\footnotetext{
${ }^{a}$ Primer names are organized in groups. Prefixes: F, forward PCR primer; R, reverse PCR primer.

${ }^{b}$ The SP6 RNA polymerase promoter sequence in primer F16 is shown in italics. Restriction sites introduced by PCR are underlined and specified in parentheses at the end of the sequence. Silent mutations within the viral sequence are shown in boldface. Fragments A through $\mathrm{F}$ correspond to the letters in Fig. 1.
} 


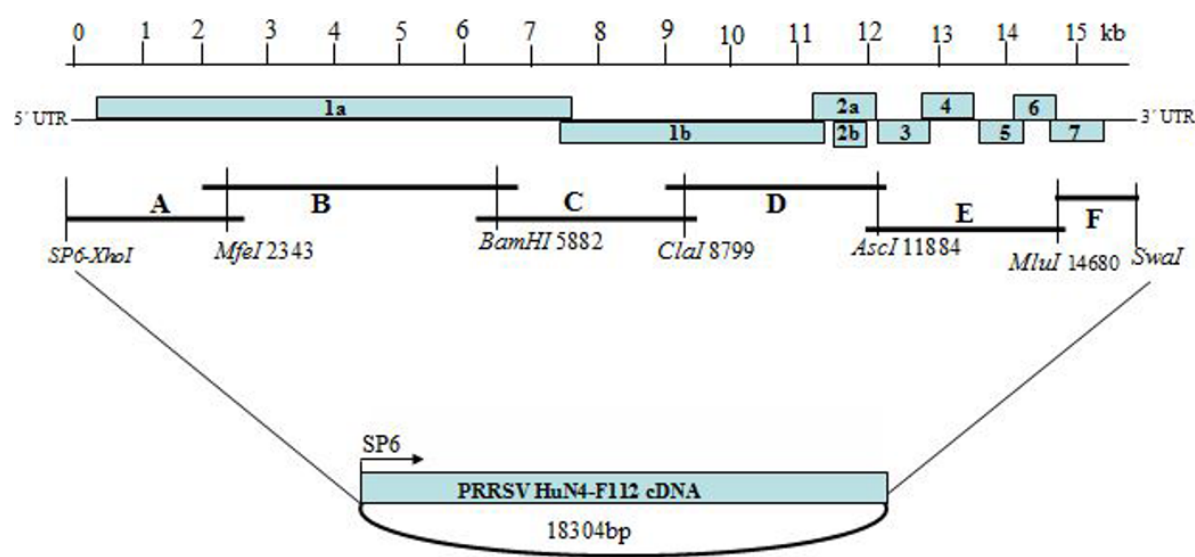

Figure 1 Strategy for the construction of the full-length cDNA clone of an attenuated live PRRSV vaccine strain, HuN4-F112. The organization of the viral genome is shown, as are the positions of the unique restriction sites used for cloning purposes. The numbers $1 a, 1 b$, $2 a, 2 b$ and 3 through 7 indicate the PRRSV open reading frames. An SP6 RNA promoter with two nontemplated G residues preceded the viral genome. The complete viral genome was divided into six fragments flanked by unique restriction sites, represented by the horizontal lines labeled A through $\mathrm{F}$. The fragments were inserted into the modified pBluescript II SK(+) vector.

\section{In vitro transcription and transfection}

The full-length cDNA clone was linearized by cleavage with restriction enzyme SwaI, which cuts downstream of the poly (A) tail. Linearized plasmid DNA was used for in vitro transcription of capped RNA with the mMessage High Yield Capped RNA Transcription kit (Ambion) according to the manufacturer's instructions and including treatment of the RNA with DNase to remove input plasmid. The transcribed RNA was purified with a MEGA clear kit (Ambion) following the manufacturer's instruction and quantified by spectrophotomery. The synthetic RNA was transfected into BHK-21 cells using DMRIE-C reagent (Invitrogen) according the protocol recommended by the manufacturer. To rescue the virus, cell culture supernatant obtained $24 \mathrm{~h}$ post-transfection was serially passaged on MARC-145 cells. Rescue of infectious virus was confirmed by indirect immunofluorescence assay (IFA) using anti-N monoclonal antibody N3H2 [16].

\section{Discrimination between the cloned virus and the parental virus}

Viral RNAs were extracted from cell culture supernatants of the infected cells by using an RNeasy Plus Mini kit (QIAGEN). RT-PCR was performed with the pairs of primer: JD1, 5'-CACAGCTCCACAGAAGGTGC-3' and JD2, 5'-TAACAGCTTTTCTGCCACCC-3'. The amplified product was digested by $M l u$ I to check the genetic marker engineered into the cloned virus.

In vitro stability and growth kinetics of the rescued virus To assess the replication stability of the rescued viruses, the cloned viruses were serially passaged (F4 to F6) in MARC-145 cells, and the CPE and the presence of genetic markers in the viruses were observed. Virus titers in cell cultures for each of passage were determined by a microtitration infectivity assay and recorded as TCID $_{50}$ per milliliter by using the Reed-Muench method. Briefly, cells were prepared in 96-well plates and inoculated with virus suspensions $(100 \mu \mathrm{L} /$ well $)$, which were prepared by serial 10 -fold dilution. After absorption for $1 \mathrm{~h}$ at $37^{\circ} \mathrm{C}$, the liquids in the wells were removed, and DMEM with $5 \%$ FBS was added to the wells. Plates were incubated for an additional 72 to 96 h; virus titers were determined by the presence of a visible CPE.

Growth kinetics studies were performed by infecting MARC-145 cells with the rescued virus and parental virus at an MOI of 0.1 . After $1 \mathrm{~h}$ of virus adsorption, cells were washed and incubated in DMEM with 3\% FBS at $37^{\circ} \mathrm{C}$. The virus-infected supernatants were collected at various time points and viral titers were determined and expressed as $\mathrm{TCID}_{50}$ per milliliter.

\section{Results}

Sequence determination of the PRRSV HuN4-F112 genome

The PRRSV HuN4-F112 strain genome was determined to be 15352 nucleotides in length. The genome contain nine deduced open reading frames (ORF) which is flanked by a 5'untranslated region (5'UTR) of 189 nt and a 3'untranslated region (3'UTR) of 150 nt excluding the poly(A) tail, respectively.

\section{Construction of full-length cDNA clone of HuN4-F112 and determination of its infectivity}

The plasmid pHuN4-F112 that contained the full-length cDNA of the entire viral genome of the HuN4-F112 was linearized by digestion with $S w a \mathrm{I}$, and used for in vitro transcription by SP6 RNA polymerase to synthesize capped 
RNAs. To recover infectious virus from the full-length cDNA clone, BHK-21 cells were transfected with the capped RNA with the transfection reagent DMRIE-C. Supernatants from the transfected BHK-21 cells obtained $24 \mathrm{~h}$ post transfection were serially passaged on Marc- 145 cells, at $72 \mathrm{~h}$ post-infection (p.i.), cytopathic effects (CPEs) was observed in cells (Figure 2). Three days following transfection of MARC-145 cells with supernatants of cells infected with cloned and parental virus, immunofluorescence assay (IFA) was performed to examine the expression of nucleocapsid (N) protein. As shown in Figure 3, the $\mathrm{N}$ proteins of both cloned and parental virus were expressed in MARC-145 cells. Thus, our results show that infectious virus was rescued from BHK-21 and MARC-145 cells transfected with capped in vitro-transcribed RNA.

\section{Discrimination between cloned virus and parental virus}

To differentiate the rescued viruses and parental viruses, a genetic marker, MluI site at nt 14680 , was engineered into the genome length cDNA. As expected, the RT-PCR fragment derived from the cloned virus was cleaved by $M l u \mathrm{I}$, generating two fragments of $360 \mathrm{bp}$ and $92 \mathrm{bp}$ (Figure 4). In contrast, the PCR amplification products derived from the parental isolate was not cleaved by MluI (Figure 4).

\section{Characterization of the cloned virus}

Growth kinetics studies were performed to compare the growth of rescued virus and parental virus in MARC-145 cells. Results (Figure 5) show that growth kinetics of rescued virus and parental virus was not significantly different from each other and that titers peaked at $48 \mathrm{~h}$ pi for both viruses. The result indicates that the rescued virus possesses growth characteristics similar to the parental virus.

\section{Discussion and conclusions}

In this study, we described the complete nucleotide sequence of the viral genome and the construction of infectious cDNA clone of HuN4-F112, a live-attenuated
$\mathbf{A}$

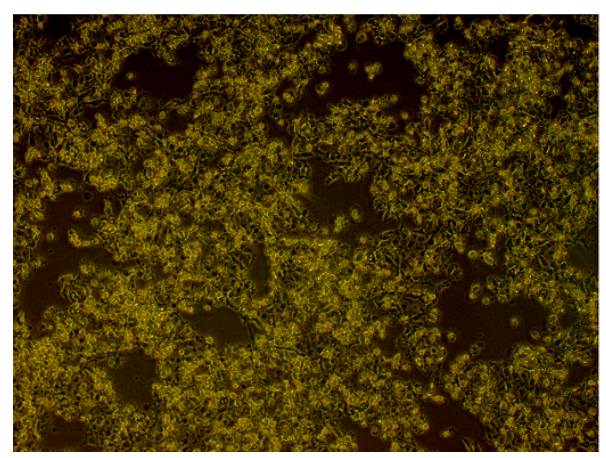

B

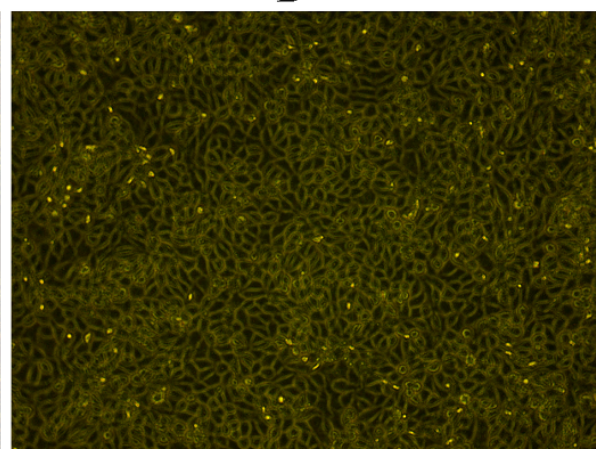

Figure 2 Cytopathic effect (CPE) of the cloned virus in Marc-145 cells. Supernatants from the transfected BHK-21 cells obtained $24 \mathrm{~h}$ posttransfection were serially passaged on Marc-145 cells, at $72 \mathrm{~h}$ post-infection (p.i.), CPE was observed in rescued virus (A), while mock-infected cells remained normal (B).

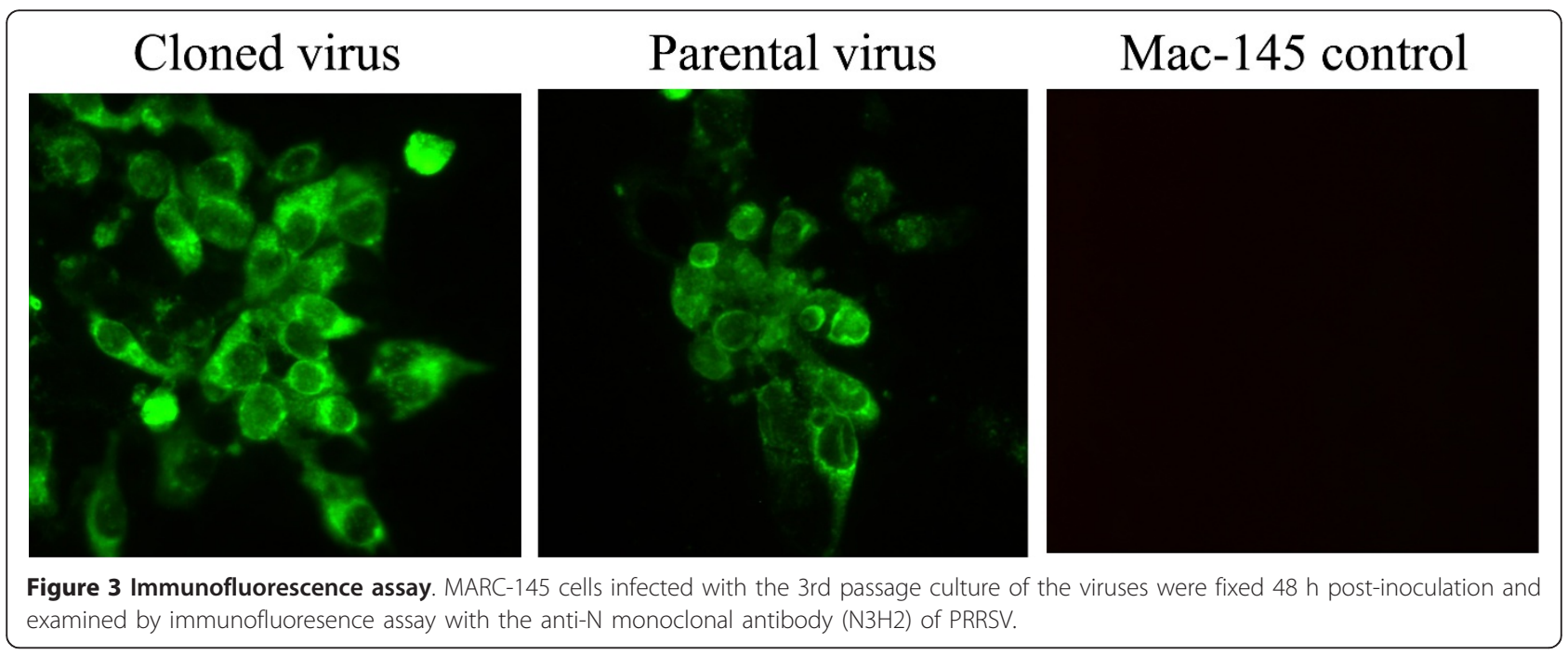




\section{Parental Cloned}

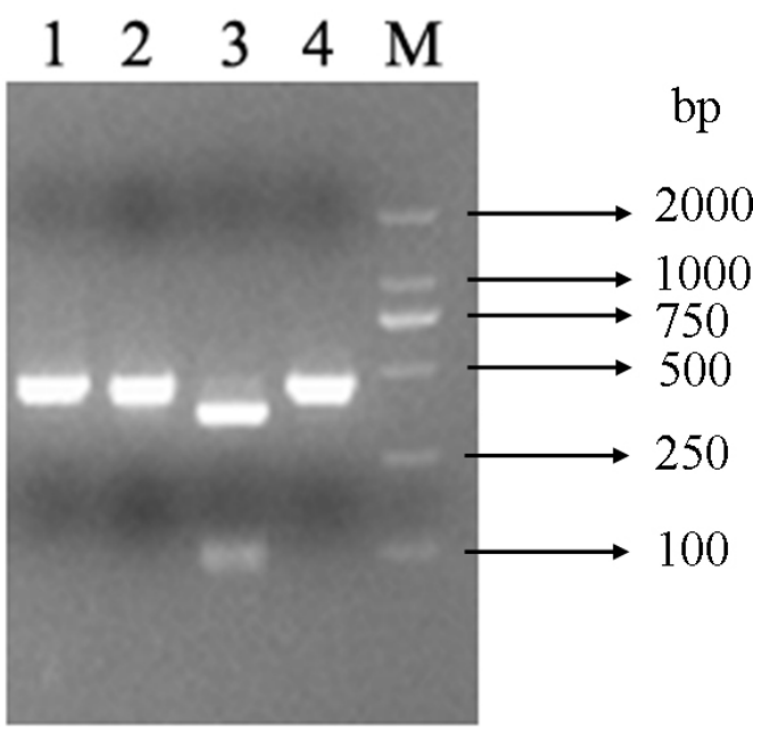

Figure 4 Differentiation between cloned virus and parental HuN4-F112 strain. A Mlul restriction site was introduced in the fulllength cDNA clone of HuN4-F112 to allow discrimination between cloned virus (tagged with the M/ul site) and parental virus (lacks the Mlul site). The presence of a Mlul restriction site resulted in fragments of $460 \mathrm{bp}$ and $92 \mathrm{bp} .1$ and 4 were RT-PCR fragments undigested, 2 and 3 were RT-PCR fragments digested by M/ul.

\section{Growth kinetics of cloend and parental viruses}

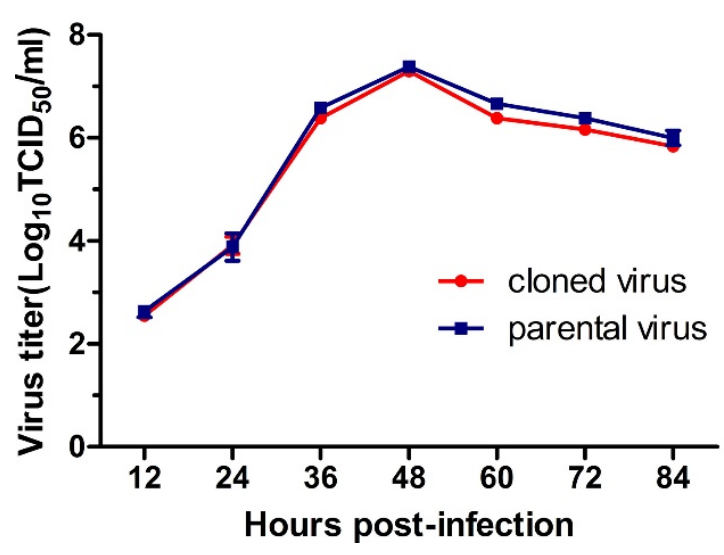

Figure 5 Growth kinetics of cloned and parental virus. The growth curves of the rescued virus and parental virus were drawn by assaying the viral titers of the supernatants obtained from $12 \mathrm{~h}$ to $84 \mathrm{~h}$ postinfection by using microtitration infectivity assays. Data are calculated and shown as means \pm standard deviations (error bars) from three independent trials.
North-American-type PRRSV vaccine strain. Transfection of capped in vitro transcripts, derived from this clone, into BHK-21 cells and Marc-145 cells led to efficient recovery of infectious virus from cultured cells. Indeed, at 3 days post-transfection, CPE was observed in transfected cells. The rescued virus displayed similar growth properties to its parent virus in MARC-145 cells. Detection of the $M l u \mathrm{I}$ genetic marker indicates that the rescued virus remained genetically stable during passage in cells.

Since May 2006, atypical PRRS (so-called porcine high fever syndrome (PHFS) in china) was pandemic in china. Several studies have confirmed that the causative agent of this outbreak was highly pathogenic PRRSV (HPPRRSV), several HP-PRRSV strains were isolated and their reverse genetic systems were developed by different labs $[13,17-20]$. We also isolated an atypical PRRS strain named $\mathrm{HuN} 4$ and attenuated this strain in vitro by passaging on Marc-145 cells, the attenuated strain was designated HuN4-F112 (112 ${ }^{\text {th }}$ passage). Study shows that HuN4-F112 was sufficiently attenuated and antigenic enough to confer protection against a lethal wild-type challenge $[10,15]$.

Sequence alignment between HuN4-F112 and its parental virulent isolate HuN4 showed that both the 5'UTR and 3'UTR are identical and mutations are presented in both nonstructural and structural protein regions. We surmise that the basis of PRRSV attenuation may be multifactor. A recent study also confirmed this by constructing of a series of chimeric viruses where specific genomic regions of a highly virulent PRRSV infectious clone (FL12) were replaced with their counterparts of an attenuated vaccine strain PrimePac. Results indicate that NSP3-8 and GP5 are the location of major virulence determinants, while other virulence determinants may also be contained in NSP1-3, NSP10-12 and GP2 [21]. The availability of the complete genome sequence and infectious clone of the virulence-attenuated strain HuN4F112 would make it possible to use a comparative virology approach to dissect the virulence determinants of PRRSV. This approach will likely yield new information on PRRSV virulence regulation and virus-host interaction in the future.

In conclusion, we rescued virus from an infectious cDNA clone of attenuated vaccine. It is possible in the future that a new attenuated PRRSV vaccine with broader specificity and good immunogenicity can be designed in vitro via an infectious cDNA clone platform coupled with validated information on virulence determinants.

\section{Acknowledgements}

The study was supported by grants from NSFC-Guangdong Joint Foundation (U0931003), the Ministry of Agriculture of China (No. 2009ZX08010-022B), International Sci \& Tech Cooperation Program 
(2010DFB33920) and the Excellent Scientist Program of Shanghai (09XD1405400).

\section{Authors' contributions}

GT and YZ have been involved in conception and design of the study, and revising the manuscript critically; SZ has been involved in design of this study, performed the study and collected data, and prepared the first draft of the manuscript; YF participated in design of the study. GL, LY and HY participated in data analysis. All authors read and approved the final manuscript.

\section{Competing interests}

The authors declare that they have no competing interests.

Received: 17 June 2011 Accepted: 19 August 2011

Published: 19 August 2011

\section{References}

1. Keffaber KK: Reproductive failure of unknown etiology. American Association of Swine Practitioners News 1989, 1:1-9.

2. Meulenberg JJ, Hulst MM, de Meijer EG, Moonen JM, den Besten, De Kluyver EP, Wensvoort G, Moormann RJM: Lelystad virus, the causative agent of porcine epidemic abortion and respiratory syndrome (PEARS), is related to lactate dehydrogenase-elevating virus and equine arteritis virus. Virology 1993, 192:62-72.

3. Christianson WT: Experimental reproduction of swine infertility and respiratory syndrome in pregnant sows. Am J Vet Res 1992, 53:485-488.

4. Dee SA, Joo HS, Polson DD, Marsh WE: Evaluation of the effects of nursery depopulation of the profitability of 34 pig farms. Vet Rec 1997, 140:498-500.

5. Albina E: Epidemiology of porcine reproductive and respiratory syndrome (PRRS): an overview. Vet Microbiol 1997, 55:309-316.

6. Cavanagh D: Nidovirales: a new order comprising Coronaviridae and Arteriviridae. Archi Virol 1997, 142:629-633.

7. Snijder EJ, Meulenberg JJ: The molecular biology of arteriviruses. J Gen Virol 1998, 79:961-979.

8. Nelsen CJ, Murtaugh MP, Faaberg KS: Porcine reproductive and respiratory syndrome virus comparison: divergent evolution on two continents. J Virol 1999, 73:270-280

9. Stadejek T, Stankevicius A, Storgaard T, Oleksiewicz MB, Belak S, Drew TW, Pejsak Z: Identification of radically different variants of porcine reproductive and respiratory syndrome virus in Eastern Europe: towards a common ancestor for European and American viruses. J Gen Virol 2002, 83:1861-1873.

10. Tong GZ, Zhou YJ, Hao XF, Tian ZJ, Qiu HJ, Peng JM, An TQ: Identification and molecular epidemiology of the very virulent porcine reproductive and respiratory syndrome virus emerged in China. Chinese Journal of Preventive Veterinary Medicine 2007, 29:323-326.

11. Tian KG, Yu XL, Zhao TZ, Feng YJ, Cao Z, Wang CB, Hu Y, Chen XZ, Hu DM, Tian XS, Liu D, Zhang $S$, Deng $X Y$, Ding $Y Q$, Yang $L$, Zhang $Y X$, Xiao $H X$, Qiao MM, Wang B, Hou LL, Wang XY, Yang XY, Kang LP, Sun M, Jin P, Wang SJ, Kitamura Y, Yan JH, Gao GF: Emergence of fatal PRRSV variants: unparalleled outbreaks of atypical PRRS in China and molecular dissection of the unique hallmark. PLOS ONE 2007, 2:e526.

12. Li YF, Wang XL, Bo KT, Wang XW, Tang B, Yang BS, Jiang WM, Jiang $P$ : Emergence of a highly pathogenic porcine reproductive and respiratory syndrome virus in the Mid-Eastern region of China. Vet J 2007, 174:577-584

13. Lv J, Zhang J, Sun Z, Liu W, Yuan SS: An infectious cDNA clone of a highly pathogenic porcine reproductive and respiratory syndrome virus variant associated with porcine high fever syndrome. J Gen Virol 2008, 89:2075-2079.

14. Zhou L, Zhang JL, Zeng JW, Yin S, Li YH, Zheng LY, Guo X, Ge XN, Yang HC: The 30 amino acids deletion in Nsp2 of highly pathogenic porcine reproductive and respiratory syndrome virus emergence in China is not related to its virulence. J Virol 2009, 83:5156-5167.

15. Tian ZJ, An TQ, Zhou YJ, Peng JM, Hu SP, Wei TC, Jiang YF, Xiao Y, Tong GZ: An attenuated live vaccine based on highly pathogenic porcine reproductive and respiratory syndrome virus (HP-PRRSV) protects piglets against HP-PRRS. Vet Microbiol 2009, 138:34-40.
16. Zhou YJ, Hao XF, Tian ZJ, Tong GZ, Yoo D, An TQ, Zhou T, Li GX, Qiu HJ, Wei TC, Yuan XF: Highly virulent porcine reproductive and respiratory syndrome virus emerged in China. Transbound Emerg Dis 2008, 55:152-164.

17. Liu G, Nielsen J, Oleksiewicz MB, Botner A, Storgaard T, Faaberg KS: Generation of an Infectious Clone of VR-2332, a Highly Virulent North American-Type Isolate of Porcine Reproductive and Respiratory Syndrome Virus. J Virol 2003, 77:3702-3711.

18. Meulenberg JJ, Bos-de Ruijter N, Van de Graaf R, Wensvoort G, Moormann M: Infectious transcripts from cloned genomic-length CDNA of porcine reproductive and respiratory syndrome virus. J Virol 1998, 72:380-387.

19. Fang Y, Rowland RR, Roof M, Lunney JK, Christopher-Hennings J, Nelson EA A full-length cDNA infectious clone of North American type 1 porcine reproductive and respiratory syndrome virus: expression of green fluorescent protein in the Nsp2 region. J Virol 2006, 80:11447-11455.

20. Lu Z, Kutish G, Galeota J, Osorio FA, Pattnaik AK: A highly pathogenic porcine reproductive and respiratory syndrome virus generated from an infectious CDNA clone retains the in vivo markers of virulence and transmissibility characteristics of the parental strain. Virology 2004, 325:308-319.

21. Kwon B, Ansari $I H$, Pattnaik AK, Osorio FA: Identification of virulence determinants of porcine reproductive and respiratory syndrome virus through construction of chimeric clones. Virology 2008, 380:371-8.

\section{doi:10.1186/1743-422X-8-410}

Cite this article as: Zhang et al:: Generation of an infectious clone of HuN4-F112, an attenuated live vaccine strain of porcine reproductive and respiratory syndrome virus. Virology Journal 2011 8:410.

\section{Submit your next manuscript to BioMed Central and take full advantage of:}

- Convenient online submission

- Thorough peer review

- No space constraints or color figure charges

- Immediate publication on acceptance

- Inclusion in PubMed, CAS, Scopus and Google Scholar

- Research which is freely available for redistribution

Submit your manuscript a www.biomedcentral.com/submit
C Biomed Central 